\title{
In search of common ground
}

\author{
With the number of stem-cell lines rapidly increasing, technology developers are working to improve \\ systems for culturing and efficient differentiation - all with an eye on the clinic. Nathan Blow reports.
}

The explosion in stem-cell research that followed the isolation of human embryonic stem cells ${ }^{1}$ in 1998 has seen the number of cell lines available to researchers increase dramatically. This burst may be due to the fact that embryonic stem cells are pluripotent - having the potential to generate all adult and embryonic cell types - so there are exceptional possibilities for their use in medicine.

Since 1998, about 200 embryonic stem-cell lines have been derived along with many more adult stem-cell lines. Supporting all these stem cells are many ways to help propagation and differentiation. So it should come as no surprise to learn that there are no standard culture conditions for stem cells. "People sometimes bristle at the word standard," says Derek Hei, director of the US National Stem Cell Bank (NSCB) in Madison, Wisconsin. "In the stem-cell community there is not really a standard culture method."

But 'standard' should not be an alarming word. "In talking to stem-cell researchers, we found that they are really looking for standardization of culture methods," says Tori Richmond, strategic initiatives specialist at Thermo Fisher Scientific in Waltham, Massachusetts. Arriving at any such standard would require evaluation of all available cell lines on every culturing system - a gargantuan task. But Martin Pera, director of the Institute for Stem Cell and Regenerative Medicine of the University of Southern California in Los Angeles, suspects that larger groups, such as the International Stem Cell Initiative, might tackle the standardization issue in the near future. "I am hoping that within the next couple of years we will arrive at one or two cellculture platforms that everyone can use."

\section{The cell in a haystack}

Several companies and stem-cell banks are now providing a range of older and more recently derived embryonic stem-cell lines to researchers, which has provided a greater understanding of the properties of these cells.

Ethical concerns regarding the use of embryonic stem cells led the US government to establish a list of 21 embryonic stem-cell lines that can be used for research funded by federal sources. To this end, the NSCB was established in 2005 to characterize, hold and distribute these approved human embryonic stem-cell lines. For each of these lines, the bank is currently performing extensive testing, says Hei, including characterization of gene expression profiles, karyotype stability, and other standard embryonic stem-cell assays such as

stem-cells lines derived at the company. Cellartis in Gothenburg, Sweden, and Millipore in Billerica, Massachusetts, also provide cell lines. Cellartis is one of the largest sources for defined embryonic stem-cell lines, boasting 30 different lines that can be obtained at various stages of passage or as subclones of selected

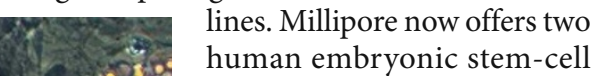
lines, human neural progenitors, as well as several mouse embryonic stem-cell lines.

\section{Search and you will find}

Other companies deriving new human embryonic stem-cell lines can also provide them to researchers. "If people request them, then yes, we will provide lines," says Robert Lanza, chief scientific officer at Advanced Cell Technology, in Worcester, Massachusetts. He says that Advanced Cell Technology also plans to give several lines to the Massachusetts stem-cell bank.

Having many different cell lines available for research might be critical to understanding the true potential of

flow cytometry for specific cellular markers of pluripotency.

At present the NSCB offers 15 of the 21 federally approved embryonic stem-cell lines to researchers. "We have set up a website and created master cell banks for each of the lines," Hei says, adding that not only is the characterization data posted online for interested researchers, but the NSCB also provides protocols for propagating and maintaining cell lines on its site. The University of Massachusetts Medical School in Shrewsbury is also expected to open a stem-cell bank to distribute embryonic stemcell lines derived by researchers within the state, including more than 30 embryonic stem-cell lines to be supplied by Harvard University, during the next year.

Similar repositories also exist outside the United States. For example, the UK Stem Cell Bank in Hertfordshire, started in 2003, and currently provides eight different human embryonic stem-cell lines. Other new stem-cell banks are on the way.

ES Cell International (ESI) in Singapore is one of a number of commercial sources for researchers looking to obtain human embryonic stem cells. It offers six of the US-approved lines, as well as several other human embryonic these cells. "It is funny, people say 'embryonic stem cells' but the cell lines all have their own behaviour," notes Lanza. Although a recent study by the International Stem Cell Initiative examining 59 human embryonic stem-cell lines found a high degree of phenotypic similarity between all lines ${ }^{2}$, some researchers have noted differences is the behaviour of the cell lines in

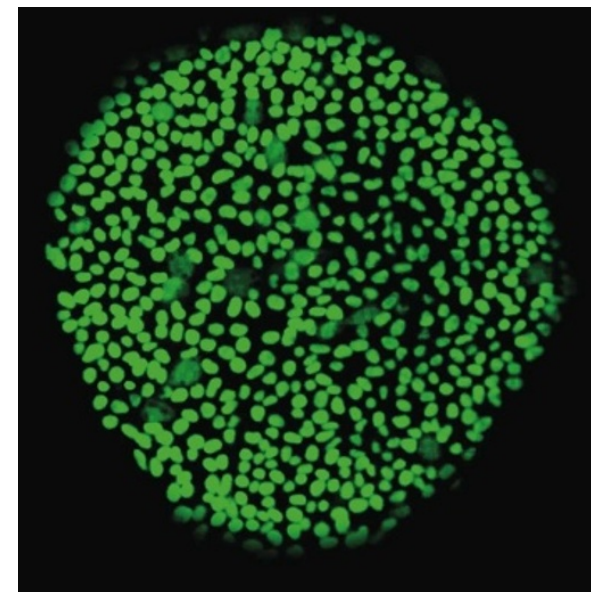

Immunochemical staining of undifferentiated human embryonic stem cells cultured on BD Matrigel Matrix in $\mathrm{mTeSR}-1$ medium. 

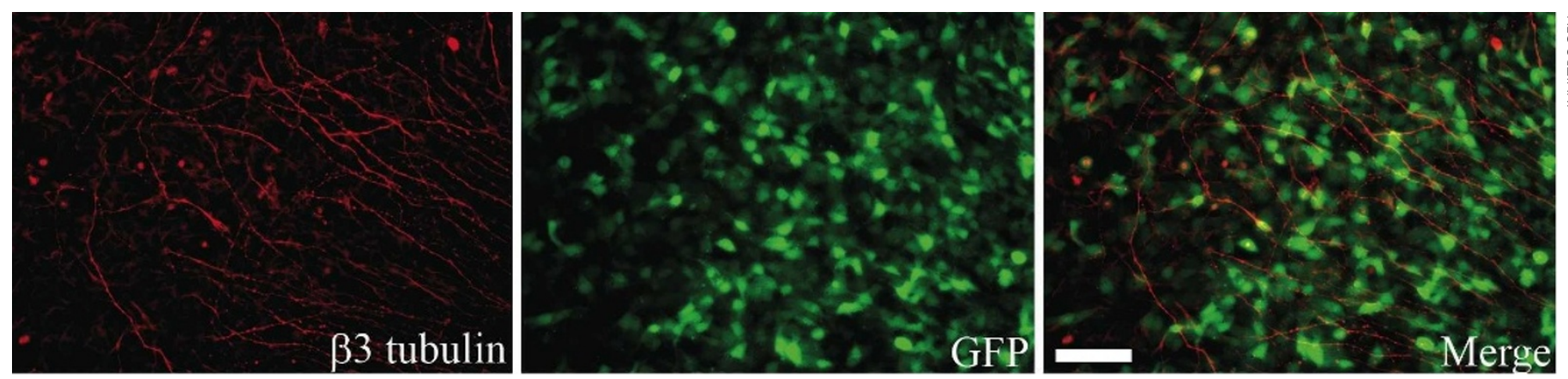

Engineered stem cells differentiated into $\$ 3$ tubulin-expressing neurons (red) while continuing to express green fluorescent protein.

culture. "Cell lines vary in how easy they are to propagate in vitro," says Pera. And Lanza notes that researchers at Advanced Cell Technology can tell embryonic stem-cell lines apart based on the behaviour of the cells in culture.

\section{The mature way to look at stem cells}

Although embryonic stem cells are pluripotent, adult stem cells are multipotent, and can only regenerate specific adult cell types in the body. And for those researchers interested in investigating the potential of adult somatic stem cells, several companies are advancing the idea of providing adult stem cells that have been qualified for culturing on specific media.

Thermo Fisher Scientific supplied media and reagents for culturing adult stem cells, but has now moved into supplying adult stem-cells lines as well. "Our strategy has not only been to create kits to support culturing and differentiation of adult stem cells, but also offer the validated stem cells themselves," says Alain Fairbank, research market manager at Thermo Fisher Scientific. The company now offers four mesenchymal (multipotent stromal cells) stem-cell lines, a haematopoietic (germinal cells from umbilical cord blood) stem-cell line, and a recently discovered multipotent cord-blood cell line that Fairbank says seems to be less restricted in its multi-lineage potential. The advantage of this approach is that all media, reagents and cultureware are certified and validated to work with these specific cell lines. "Our focus has been developing tools that are validated to work together with stem cells," Fairbank says.

Invitrogen of Carlsbad, California, is also "heavily focused on mesenchymal research", says Joydeep Goswami, the company's vicepresident of stem-cell research. "These cells are probably going to be the first non-haematopoietic stem cells to be used for treating patients." In addition to providing adult stem- cell lines, Invitrogen is also actively researching engineered stem cells. "We have been doing a lot of research to convert stem cells themselves into tools," says Goswami. He says that these engineered cell lines act as single-cell reporters providing a visual readout from a live stem cell as it differentiates to separate lineages.

But even as certain adult stem cells become more readily available with well-validated approaches to culturing and differentiation, there is still much work to be done. "Many adult stem-cell populations remain difficult to propagate and expand ex vivo," says Pera. And with the exception of mesenchymal stem cells, he says, this has not changed dramatically in recent years. Although Goswami agrees, he also points to another potential issue. "It is not that adult stem cells have not been isolated, the bigger issue is how do you get well characterized stem cells?" He notes that in some instances researchers will call

\section{BEYOND THE FLAT WORLD}

In culture, stem cells rely on signals to differentiate to other cell lineages. Although certain growth factors are known to promote some differentiation programmes, it is now becoming clear that physical interactions between cells and mechanical sensing may also help to promote differentiation. So researchers are developing a variety of three-dimensional (3D) matrices for stem-cell differentiation.

In 2006, Dennis Discher of the University of Pennsylvania in Philadelphia, and his colleagues, demonstrated the potential of the matrix alone to promote differentiation. "The idea was to use synthetic gels with a collagen monolayer to mimic the elasticities of certain tissues and see how the cells respond," says Discher. The work was done under constant serum conditions, without any discriminating soluble factors or growth factors that might promote differentiation in one direction or another. The results showed that by simply varying the elasticity of the matrix, the attached mesenchymal stem cells could undergo either neurogenesis, myogeneis or osteogenesis. They went on to show that once the cells adhere, they begin setting up the stress fibres that actively pull on the adhesions and on the matrix outside. "We showed that the cells feel the matrix and respond to it," says Discher.

Stefan Przyborski is the founder of Reinnervate in Durham, UK, the developer of a new scaffold for routine 3D cell culture. "We create ways of making the in vitro environment a more realistic environment for cell growth," he says. Reinnervate's scaffold is unique, but might seem familiar to biologists who have performed cell culture. "We created a scaffold made of polystyrene, the same material that people currently

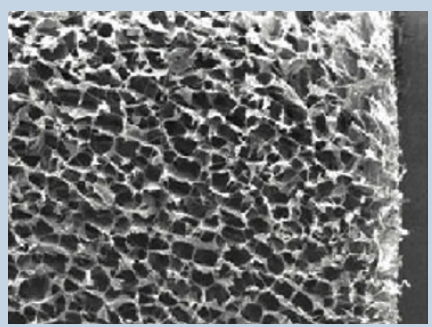

Three-dimensional matrices can promote cell differentiation.

grow their cells on." This is important because researchers know how cells respond to this material in two-dimensional applications. But he also notes that this 3D environment enhances differentiation when compared with cells cultured on twodimensional plates.

Invitrogen of Carlsbad, California has developed Algimatrix, an inert 3D scaffold made of alginate.

"The idea behind Algimatrix is that it provides a framework for cells to reside in that will enable formation of spheroidal structures in a controlled manner," says Mark Powers, a director at Invitrogen. Powers says that when embryonic stem cells form embryoid bodies, they can aggregate into very large structures where the cells on the inside can be oxygen or nutrient limited. But with Algimatrix the aggregates grow to a consistent size and not beyond the size of the pores provided by the scaffold. And since it is an inert scaffold, it actually promotes cell interactions. "A scaffold such as Algimatrix would allow a researcher to culture cells in 3D aggregates to promote differentiation."

It is becoming clear that mechanical interaction has a role in cell differentiation. "What we are really describing is a sense of touch - the cells have no eyes or ears, so they use this sense of touch to tell where they are - this is all part of sensing and responding to the environment," says Discher. N.B. 
cells a particular type of stem cell, but the next batch will have different marker profiles, indicating a different cell type.

\section{Mouseless developments}

The culturing of embryonic stem cells has often relied on the use of mouse embryonic fibroblast cells (MEFs) as 'feeder' cells in different media formulations containing serum. It is thought that the MEFs secrete factors that promote the growth of embryonic stem cells in culture. But growing embryonic stem cells using MEFs in undefined media and serum that potentially contains animal products could limit the potential of stem-cell lines for therapeutic applications.

"It all comes down to how the regulators look at these lines and how they have been handled over the years," says Bruce Davidson, chief scientific officer at ESI. It is for this reason that ESI worked to derive clinical-grade human embryonic stem cells. "We have four GMP- [good manufacturing practice] certified embryonic stem-cell lines that were developed over the past couple of years and are now ready for distribution," says Davidson. Cellartis also provides a human embryonic stem-cell line that was derived without any contact with animal products. And many more researchers and companies are trying to identify the important factors for culturing embryonic stem cells and develop robust feeder-free approaches in well-defined media.

The WiCell Research Institute in Madison, Wisconsin, has a major focus on optimizing embryonic stem-cell culture media and conditions to allow long-term culture of stem cells that do not differentiate. In recent years, researchers at WiCell have found that small changes to either media or the physiochemical conditions of the culture can have a profound effect on the viability of cells. But in 2006, WiCell researchers

\section{方}

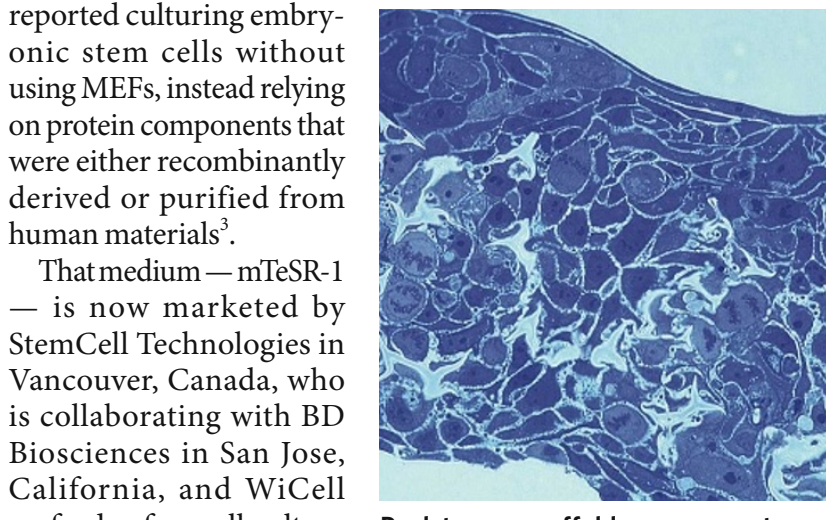

on feeder-free cell culture environments for human embryonic stem-cell culture using BD Biosciences Matrigel scaffold. "Colonies formed on Matrigel hESC-qualified matrix with mTeSR-1 media tend to be spread out and form a monolayer-like morphology, which makes passage easier and transfection more efficient," says Marshall Kosovsky, technical support manager at BD Biosciences. Unlike three-dimensional growth matrices, system, he says. where cell-cell interaction can promote differentiation, Matrigel is coated as a twodimensional scaffold and is therefore effective for long-term embryonic stem-cell culture. Although Matrigel is a solubilized preparation extracted from a mouse sarcoma - potentially raising similar issues to the use of serum Kosovsky says that they are currently working on more defined formulations of the scaffold.

Invitrogen is one of several companies moving away from culture conditions that rely on either feeder-cells or serum. "We are providing stem-cell media with a twist - it is a serum-free and defined

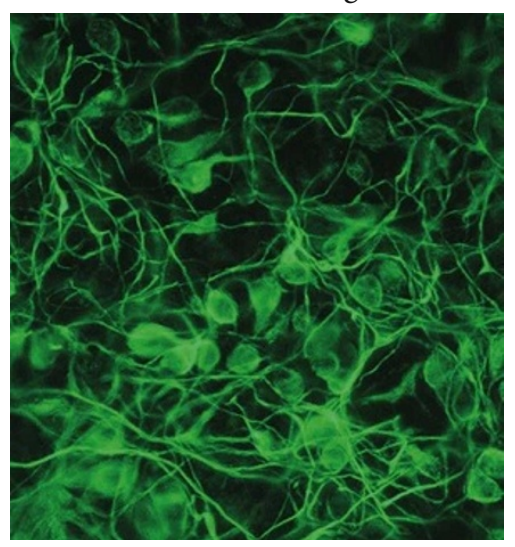

Reinnervate in Durham, UK, derives neurons from human pluripotent stem cells using synthetic compounds.
Differentiation of embryonic-stem cells or adult stem cells into particular lineages is an area of intense research for developmental biologists and researchers interested in using stem calls for therapeutic applications.

"This is where the battlefront is," says Lanza, "We need to learn how to generate different cell types." He points to haematopoetic stem cells, which give rise to all blood cell types, as an example of how difficult the task can be. In an embryo these cells start out in the yolk sac, then travel to the aortic arch, to the liver and then to the bone marrow, and at each point along that journey are being 'edumedia," says Goswami. This medium, called StemPro hESC SFM, works with 16 embryonic stem-cell lines from around the world. Millipore also offers a serum-free medium called HEScGRO, which works with a variety of embryonic stem-cell lines.

\section{Different paths}

"The field is moving towards defined media without animal components and without feeders," says Pera. But some scientists are being cautious about these culture systems. Although signs are encouraging, Pera says that the jury is still out on most of these new systems. And the NSCB is taking a careful approach to feederfree cultures. "We made the decision not to use some of the feeder-free methods that are coming out right now, but to stick at this stage to the standard MEF-based methods," says Hei. The decision was made in part to avoid forcing the research community towards feederfree methods while they are still in the early stages of development. But Hei also acknowledges that at a later date they might develop banks of cells using feederfree systems. Although some express concerns that cells cultured in feeder-free systems do not thrive as well, Goswami is confident in these new systems and says that the time has come for feeder-free, serum-free embryonic stem-cell culture. "Using our media we can get equivalent or higher numbers of cells" compared with any feeder-dependent

"We are still learning a lot about what it takes to coax a stem cell to become a particular tissue," says George Daley from Harvard Medical School in Boston, Massachusetts. cated' by their surroundings. Replicating this is not easy. Advanced Cell Technology has been working on the differentiation potential of the haemangioblast - a precursor to hematopoietic cells - that they isolated. "People have got other haemangioblast-like cell types, but these are KDR-negative and CD31-negative, so they are an earlier progenitor cell than what's been described previously, which is why they expand and do things so much better," says Lanza. Differentiating these haemangioblasts has allowed the company's researchers to generate entire tubes of red blood cells, as well as other hematopoietic lineages and endothelial cells at very high efficiencies.

Although success has also been reported in using differentiation protocols for deriving neural progenitor cells, cardiomyocytes and retinal pigment epithelium from embryonic stem cells, in most cases studies have shown that only a very small percentage of cells differentiate into a particular tissue type. Some researchers now think that multiple cells or interactions between cells and surfaces might be required to obtain a desired derivative from stem cells (see 'Beyond the flat world').

But Pera sees this as one area that is steadily progressing. "I think we are getting more and better differentiation protocols that are more defined and have better endpoints," he says. However, he does think that there is lack of comparative data on different cell lines and their abilities to differentiate using the current protocols - although the available data would indicate that there will be differences between certain cell lines in the ability to perform in specific differentiation protocols.

\section{Looking up}

The world might soon be slightly easier for researchers working on mesenchymal stemcell differentiation. Both Thermo Fisher Scientific and Invitrogen are introducing kits in the coming months for the differentiation of 
mesenchymal stem cells to adipogenic (fatproducing), osteogenic (bone-producing) and chondrogenic (cartilage-producing) lineages. These kits provide all the necessary growth factors to direct the differentiation of the mesenchymal stem cells. Millipore also offers differentiation media and kits for neural stem-cell lines and mesenchymal stem cells including a human neuronal-differentiation kit and adipogenesis and osteogenesis kits. Although these kits are available for mesenchymal stem cells, the percentages of differentiated mesenchymal cells can still be low for certain lineages.

\section{Stem cells hitting the clinic?}

Even as culture and differentiation research progresses, the race is starting to bring embryonic stem-cell therapies to the clinic. Novocell of San Diego, California, is searching for a diabetes treatment, exploring the potential of using stem cells for treatment. "Using cadaveric or even fetal cells was a real challenge because you do not have an unlimited source of these cells," says Alan Lewis, chief executive of Novocell. "While developing a delivery system, in the background we were working with embryonic stem cells to derive insulinproducing cells," he says. And that work is starting to pay off because Novocell can derive definitive endoderm from embryonic stem cells and then differentiate these to insulinproducing cells. Novocell is now working to define these insulin-producing cells, while further refining its polyethylene-glycol or PEGbased delivery vehicle that could help to avoid immune-response issues in patients.

As companies move their products closer to clinical trials, the questions on everyone's mind are when and under what conditions will the US Food and Drug Administration (FDA) fire the starter's gun? Advanced Cell Technology and Geron in Menlo Park, California, are currently in discussions with the FDA regarding clinical trials using embryonic stem cellbased therapies for the coming year.

Advanced Cell Technology has developed an application to use embryonic stemcell-derived retinal

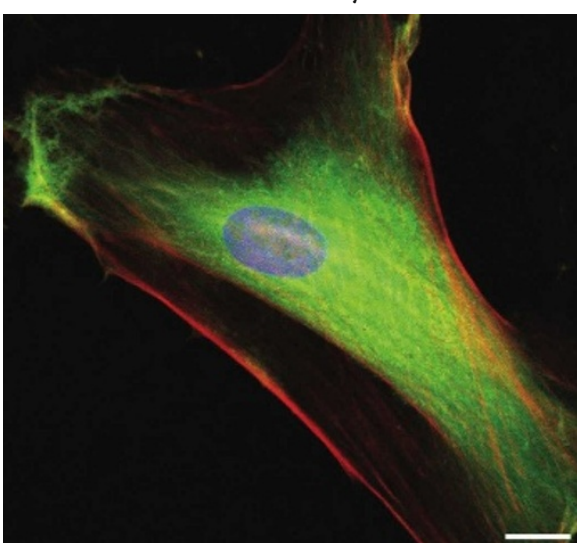

Umbilicial matters: Thermo Fisher Scientific offers adult stem-cell lines derived from Wharton's Jelly. cells thrived in culture. "It turns out that although you can derive cells on human feeders or even feeder-free with extracellular matrices, they do not thrive as well," says Lanza. And for
this reason he thinks that in the long term, when generating large batches of cells for clinical applications, the mouse feeders are currently the optimal method.

It is not yet clear what the first therapy based on embryonic stem cells to reach clinical trials will be, whether it will be using cells raised on MEFs or feeder-free media. And for some researchers it is not pigment epithelium to treat various retinal degenerative diseases such as macular degeneration and retinitis pigmentosa. The company started with this application because in addition to being able to generate large numbers of the required cells, the eye is an immune privilege site, lessening the possibility of an immune response to the cells. Geron has developed a stem cell-based treatment for acute spinal-cord injury. For both of these applications, the companies have utilized stem cells grown with mouse feeder cells. For Advanced Cell Technology this decision was based on how well the stem will be the first pluripotent cells first to reach the clinics (see 'A new path to pluripotency'). George Daley says that it is too early to tell for sure the way stem cell-based therapies will enter the clinics, but he will be watching the developments closely. "I think that it is going to be an exciting time to wait and see," he says.

Nathan Blow is the technology editor for Nature and Nature Methods.

Thomson, J. A. et al. Science 282, 1145-1147(1998).

2. Adewumi, O. et al. Nature Biotechnol. 25, 803-816 (2007)

3. Ludwig, T. E. et al. Nature Biotechnol. 24, 185-187 (2006). even clear if embryonic stem cells themselves

\section{A NEW PATH TO PLURIPOTENCY}

In November 2007, two groups headed by James Thompson at the University of Wisconsin-Madison and Shinya Yamanaka at Kyoto University in Japan made headlines when they described methods to reprogramme adult human cells to a pluripotent state. These cells, called induced pluripotent stem (iPS) cells, are genetically modified by the integration of up to four DNA-transcription factors into the adult cell genome. Soon after, in December, George Daley, of Harvard Medical School in Boston, Massachusetts, and his colleagues also demonstrated iPS cells could be generated from a wide variety of adult cells. "For any patient we can use the technique and take a skin biopsy to establish a pluripotent cell," says Daley. One of the most valuable aspects of the iPS-cell technology currently, he says, is the ability to perform disease modelling. And to take advantage of this, Daley and his colleagues

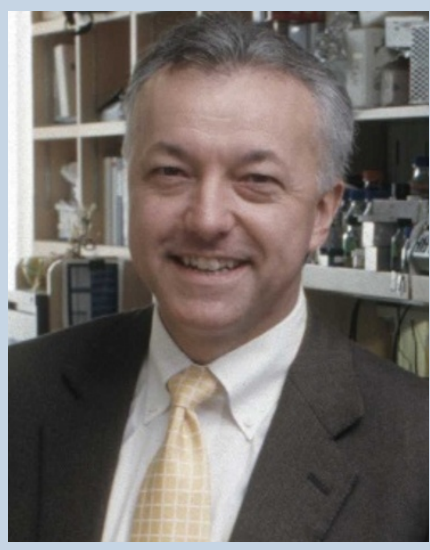

George Daley is exploring the potential of induced pluripotent cells.

are working on generating large numbers of disease specific iPScell lines.

Although an incredible step forward in stem-cell research, iPS cells are in fact at the beginning of a long road. "iPS cells as we make them today are riddled with viruses," says Daley. He says that these viruses can be mutagenic and have the potential to activate oncogenes, so at the moment iPS cells remain a research tool and not a potential therapeutic agent. But the next step for iPS cells could move them closer to therapeutic applications. "One of the next big milestones will be making these cells without the use of viruses - leaving the cells in a genetically pristine state," he says. Robert Lanza from Advanced Cell Technologies in Worcester, Massachusetts agrees and even sees routes to creating iPS cells without genetic modification. "You can potentially use fusion proteins or small molecules - there are many ways to skin the cat here."

But even if iPS cells can be created without genetic modifications, the question that researchers are asking now is, do these cells really have the same properties and potentials as embryonic stem cells? “I am very excited about iPS cells but now we need to look very carefully at the properties of these cell lines," says Martin Pera of the University of Southern California in Los Angeles. Pera says that these cells might differ in their abilities to differentiate in the same way that embryonic stem cells seem to. "If you have to make ten lines for each patient - is patient-specific therapy really realistic or will large banks of iPS cells that are tissue typed be required?"asks Pera.

Only time will tell in what directions iPS cells might be taken for basic research or for clinical applications. And while iPS-cell properties are being studied and new methods to derive these cells without genetic modifications are being created, human embryonic stem-cell research will continue. Pera says that this is the best way to proceed at the moment. "We need to move forward on both fronts." 